\title{
STEM-Based Technologies for Nano and Biological Sciences
}

\author{
X. F. Zhang* \\ * Nanotechnology System Division, Hitachi High Technologies America, Inc., 5100 Franklin Drive, \\ Pleasanton, CA 94588
}

The fast revolutions in nano and biological technologies resulted in increasingly high demand for structural and chemical analyses for nanoscale and/or light-element composition materials. Transmission electron microscopy (TEM) provides high resolution and local area imaging/electron diffraction as well as spectroscopy, therefore becomes an indispensable tool in study of nano and biological materials. However, the conventional TEM is not suitable for thick sample imaging because of the chromatic aberration of electromagnetic lenses. In addition, the small solid angle of energy-dispersive X-ray spectroscopy (EDS) attachment on a transmission electron microscope limits the sensitivity of the chemical analysis to light elements. To overcome these shortages, Hitachi has been making a continuous contribution to develop high class dedicated scanning transmission electron microscopy (STEM) instruments which are particularly useful for thick sample imaging and high sensitivity spectroscopy analyses. Liquid- and gas-STEM with nanometer to sub-nanometer resolutions, high voltage high resolution secondary electron (SE) imaging, high sensitivity STEM-EDS system, and real-time 3D STEM imaging for thick samples are among the recent progresses.

Fig. 1 shows an image of a Si particle in a 100 mbar gas environment and at a temperature of $600^{\circ} \mathrm{C}$. A window-type gas environmental-cell (E-cell) holder was used for the in situ environmental electron microscopy [1]. A liquid E-cell holder with a similar design was combined with STEM to study biological/nano systems and STEM showed clear advantages in imaging liquid samples [2].

Recently, Hitachi successfully broke the $1 \AA$ resolution for SE imaging, making true atomic resolution scanning electron microscopy (SEM) a reality for the first time [3]. Fig. 2 shows a high resolution SE image taken on a $200 \mathrm{kV}$, Cs-corrected dedicated STEM system (HD-2700C). The $0.14 \mathrm{~nm}$ separation between the neighboring Si columns ( $\mathrm{Si}$ dumbbells) clearly demonstrates the unprecedented SE image resolution. This technology has been used for biological and nanomaterial samples to study surface structures [3-5].

Combining the advantages of STEM for thick-sample imaging with a $360^{\circ}$-view specimen holder, biological whole cell samples with sample thicknesses ranging from sub-micron to more than 10 microns were imaged to reveal structural information in 3D space. Fig. 3 is an example in which a $15 \mu \mathrm{m}$-thick epoxy-embedded yeast whole cell sample was viewed from the opposite directions [6].

\section{Acknowledgements}

The author is grateful to Dr. Niels De Jonge and Dr. Jane Howe of Oak Ridge National Laboratory, and Dr. Jinsong Wu of NURANCE, Northwestern University for providing experimental data and associated discussions.

\section{References}

[1] T. Yaguchi et al., Microsc. Microanal. 16 (2010) 302. 
[2] D.B. Peckys et al., Plos One 4 (2009) e8214.

[3] Y. Zhu et al., Nature Mater. 8 (2009) 808.

[4] J.S. Wu et al., Microsc. Microanal., to be published.

[5] J. Y. Howe et al., Microsc. Microanal. 16 (2010) 312.

[6] T. Kamino et al., J. Electron Microsc. 53 (5) (2004) 563.

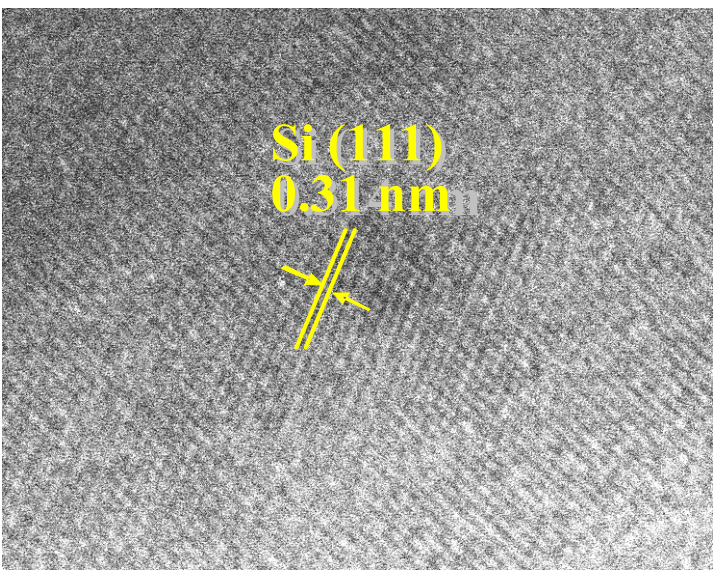

Fig. 1: TEM image of a $\mathrm{Si}$ particle in an window-type gas E-cell. The sample was heated to $600^{\circ} \mathrm{C}$ in a 100 mbar gas pressure. Si (111) lattices with a $0.31 \mathrm{~nm}$ spacing are clearly seen. A $300 \mathrm{kV} \mathrm{H}-9500$ microscope was used.

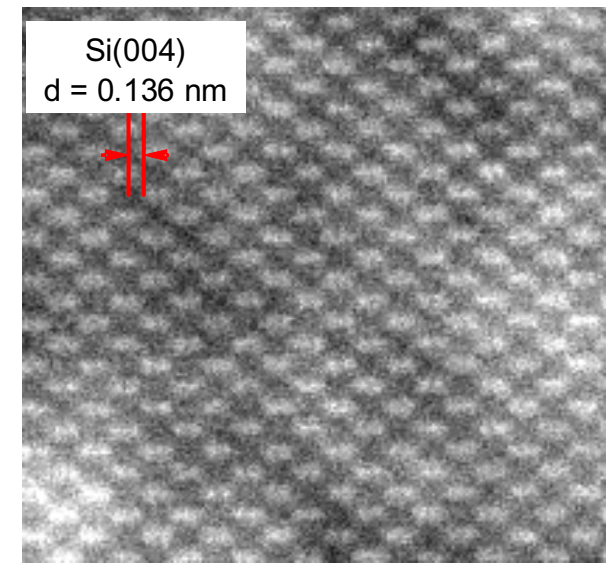

Fig. 2: SE image along the Si [110] direction. A $200 \mathrm{kV}$ aberration-corrected HD-2700C STEM system with an SE detector was used and the electron probe was $<0.1 \mathrm{~nm}$ in diameter.

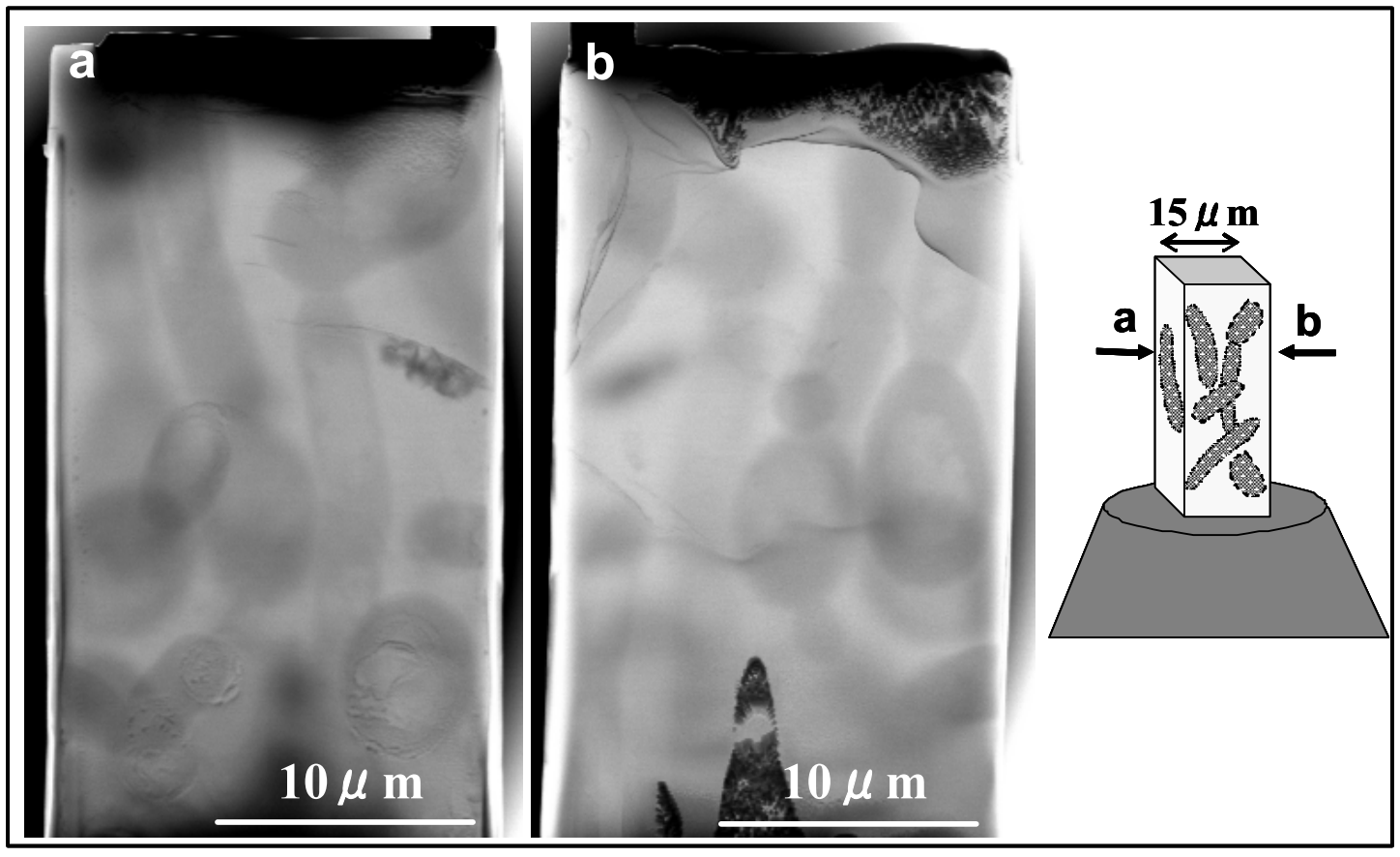

Fig. 3: STEM bright-field images of a $15 \mu \mathrm{m} \times 15 \mu \mathrm{m}$-thick pillar shaped epoxy sample containing yeast whole cells. Using a Hitachi $360^{\circ}$-view tomography holder, the sample is observed from the opposite directions (Side $\mathbf{a}$ and Side b). 